\section{Response to: 'Correspondence on 'Increased risk of systemic lupus erythematosus in patients with autoimmune haemolytic anaemia" by Huang and Zhang}

We thank Huang and Zhang $^{1}$ for their interest in our article entitled 'Increased risk of systemic lupus erythematosus (SLE) in patients with autoimmune hemolytic anaemia (AIHA): a nationwide populationbased cohort study'. ${ }^{2}$ In our article, we used nationwide longitudinal population-based data to explore the incidence of systemic lupus erythematosus (SLE) in patients with autoimmune haemolytic anaemia (AIHA) and demonstrated that patients with AIHA are at a very high risk of incidental SLE. ${ }^{2}$ Huang and $\mathrm{Zhang}^{1}$ have put forward valuable comments and opinions on our article.

First, Huang and Zhang ${ }^{1}$ proposed that patients with childhoodonset SLE may be more likely to develop AIHA than adulthood-onset patients, and suggested that we describe the incidence of AIHA in patients with SLE of different age groups. Given that it is impossible to provide such information using our original data, we conducted another nationwide cohort study to investigate the risk of AIHA in patients with SLE stratified by age groups (ie, $<18$ years and $\geq 18$ years). Using the 1997-2013 Taiwanese National Health Insurance Research Database (NHIRD), we identified 6781 newly diagnosed patients with SLE from 2006 to 2013 and randomly selected 130340 age, sex-matched (1:20) individuals without SLE from one million representative populations. From the cohort, we used propensity score matching (PSM) (1:2) for age, sex, comorbidities and potential confounders (online supplemental table 1). Before PSM, 123 (1.9\%) patients with SLE and $1(0.001 \%)$ individual without SLE had a history of AIHA ( $\mathrm{p}<0.001)$. After excluding those with a history of AIHA, the incidence rates with 95\% CIs of AIHA were 26.82 (26.80 to 26.83 ) cases per 100000 person-months in patients with SLE and $0.046(0.045$ to 0.046$)$ individuals without SLE (incidence rate ratio $88.68,95 \%$ CI 185.81 to 1865.06 ) (table 1 ). After adjusting for potential confounders, including demographic variables, medical use and comorbidities, the risk of AIHA was markedly increased in patients with SLE (adjusted HR 550.63, 95\% CI 172.64 to 1756.25) (online supplemental table 2). However, age was not associated with the risk of AIHA. Among patients with SLE before PSM, the incidence of AIHA was not significantly different between patients with childhood-onset SLE and patients with adulthood-onset SLE (table 2).

Second, Huang and Zhang ${ }^{1}$ also suggest that comparison be made on the incidences of SLE between patients with AIHA with idiopathic thrombocytopenic purpura (ITP), patients with AIHA without ITP and patients with ITP without AIHA. We found that of the 1:20 age-matched and sex-matched patients with AIHA $(n=731), 56(7.66 \%)$ had a history of ITP and 675 (92.34\%) had no comorbid ITP. The incidences of SLE were 383.15 cases per 100000 person-months and 404.47 cases per 100000 person-months in patients with AIHA with and without ITP, respectively (incidence rate ratio $0.95,95 \%$ CI 0.44 to 2.03 ). Among ITP patients without AIHA, Zhu et $a l^{3}$ recently reported that the incidence of SLE was 52.45 cases per 100000 person-months using the NHIRD. Also, we agree with Huang and Zhang $^{1}$ that both AIHA and ITP are associated with antiphospholipid antibodies. ${ }^{4}$ Unfortunately, the NHIRD lacks laboratory data. Therefore, future human and animal studies were warranted to elucidate the mechanistic role of antiphospholipid antibodies on the development of AIHA and ITP.

Finally, Huang and Zhang ${ }^{1}$ were concerned with the validity of AIHA diagnosis by ICD-9 code and suggested checking the autoantibodies to confirm AIHA diagnosis. Given that the NHIRD lacks autoantibodies data, we only considered patients whose AIHA diagnosis was made during hospitalisation but not in outpatient visits only, as patients with AIHA, to improve the validity of AIHA

Table 1 Incidence of AIHA in the study groups before and after PSM

\begin{tabular}{|c|c|c|c|c|}
\hline & \multicolumn{2}{|c|}{ Before PSM (1:20 age-sex matching) } & \multicolumn{2}{|l|}{ 1:2 PSM } \\
\hline & Non-SLE & SLE & Non-SLE & SLE \\
\hline \multicolumn{5}{|l|}{ All } \\
\hline $\mathrm{n}$ & 130339 & 6394 & 11738 & 5869 \\
\hline Follow-up (person-months) & 6586011 & 290881 & 594290 & 266895 \\
\hline SLE & 3 & 78 & 1 & 70 \\
\hline Incidence rate* $(95 \% \mathrm{Cl})$ & $0.046(0.045-0.046)$ & 26.82 (26.80 to 26.83$)$ & $0.17(0.17$ to 0.17$)$ & $26.23(26.21$ to 26.25$)$ \\
\hline Crude relative risk $(95 \% \mathrm{Cl})$ & Reference & 588.68 (185.81 to 1865.06$)$ & Reference & 155.87 (21.65 to 1122.10$)$ \\
\hline Adjusted HR $(95 \% \mathrm{Cl})$ & Reference & $550.63(172.65$ to 1756.25$) \dagger$ & Reference & $149.24(20.76$ to 1072.90$) \ddagger$ \\
\hline \multicolumn{5}{|l|}{ Age $<18$ years } \\
\hline $\mathrm{n}$ & 15440 & 749 & 1396 & 698 \\
\hline Follow-up (person-months) & 842289 & 38137 & 76401 & 35421 \\
\hline SLE & 0 & 12 & 0 & 11 \\
\hline Incidence rate ${ }^{*}(95 \% \mathrm{Cl})$ & 0 & 31.47 (31.41 to 31.52$)$ & 0 & $31.06(31.00$ to 31.11$)$ \\
\hline Crude relative risk $(95 \% \mathrm{Cl})$ & Reference & Cannot estimate & Reference & Cannot estimate \\
\hline Adjusted HR $(95 \% \mathrm{Cl})$ & Reference & Cannot estimate & Reference & Cannot estimate \\
\hline \multicolumn{5}{|l|}{ Age $\geq 18$ years } \\
\hline $\mathrm{n}$ & 114899 & 5645 & 10342 & 5171 \\
\hline Follow-up (person-months) & 5743721 & 252744 & 517888 & 231475 \\
\hline SLE & 3 & 66 & 1 & 59 \\
\hline Incidence rate ${ }^{*}(95 \% \mathrm{Cl})$ & $0.0522(0.0520$ to 0.0524$)$ & 26.11 (26.09 to 26.13$)$ & $0.19(0.19$ to 0.19$)$ & $25.49(25.47$ to 25.51$)$ \\
\hline Crude relative risk $(95 \% \mathrm{Cl})$ & Reference & 499.96 (157.20 to 1590.12$)$ & Reference & 132.00 (18.29 to 952.76$)$ \\
\hline Adjusted HR (95\% CI) & Reference & $460.24(143.69$ to 1474.12$) \dagger$ & Reference & $126.02(17.47$ to 909.10$) \ddagger$ \\
\hline
\end{tabular}

*Incidence rate, cases per 100000 person-months.

tCox proportional hazard regressions for estimation of HR on AIHA with SLE exposure adjusted for demographic variables, medical use and comorbidities at baseline.

$\neq$ Conditional Cox model for estimation of HR on AIHA with SLE exposure alone.

AIHA, autoimmune haemolytic anaemia; PSM, propensity score matching; SLE, systemic lupus erythematosus. 


\begin{tabular}{lll}
\hline & Table 2 & Incidence of AlHA in patients with SLE before PSM \\
\hline & \multicolumn{2}{l}{ Before PSM (1:20 age-sex matching) } \\
\cline { 2 - 3 } & $<18$ years & $\geq 18$ years \\
\hline $\mathrm{n}$ & 749 & 5645 \\
Follow-up person-months & 38137 & 252744 \\
SLE & 12 & 66 \\
Incidence rate* $(95 \% \mathrm{Cl})$ & $31.47(31.41$ to 31.52$)$ & $26.11(26.09$ to \\
& & $26.13)$ \\
Crude relative risk $(95 \% \mathrm{Cl})$ & Reference & $0.83(0.45$ to 1.54$)$ \\
Adjusted HRt $(95 \% \mathrm{Cl})$ & Reference & $1.02(0.52$ to 1.97$)$ \\
\hline
\end{tabular}

*Incidence rate, cases per 100000 person-months.

tCox proportional hazard regressions for estimation of HR on SLE with AIHA exposure adjusted for demographic variables, medical utilisation and comorbidities at baseline.

AlHA, autoimmune haemolytic anaemia; PSM, propensity score matching; SLE, systemic lupus erythematosus.

diagnosis. This is also the reason why the case number of patients with AIHA in a nationwide dataset was relatively small, leading to a wide range of $95 \% \mathrm{CI}$ of $\mathrm{HR}$. We also acknowledge that the extremely high HR of SLE risk in patients with AIHA compared with individuals without AIHA can be explained, at least in part, that some patients with AIHA were actually in the early stage of SLE. We tried to improve the validity of AIHA diagnosis by the exclusion of those $(n=36)$ who did not receive corticosteroid or immunosuppressant therapy withing 6 months after AIHA diagnosis and found a consistent result using the age-matched and sex-matched populations (adjusted HR 151.11, 95\% CI 92.46 to 246.95). Furthermore, Huang and Zhang ${ }^{1}$ suggested stratified analyses based on different time intervals to investigate potential time-varying effects. According to the Kaplan-Meier curves that displayed the cumulative incidence of SLE, we examined the risks of SLE associated with AIHA during the time intervals within the first 2 years and after 2 years of follow-up. We found that in the propensity score-matched population, the HRs for SLE development were 202.97 (95\% CI 28.28 to 1456.57 ) and 47.11 (95\% CI 6.25 to 355.25 ) during the first 2 years and after 2 years, respectively.

In conclusion, patients with AIHA had increased risk of SLE particularly in the first 2 years. The risk did not differ between patients with AIHA with and without a history of ITP. We also found a markedly increased risk of AIHA in patients with SLE, and the risk was not significantly different between patients with childhoodonset SLE and patients with adulthood-onset SLE. We suggested that clinicians conduct comprehensive assessment regarding SLE-related clinical and laboratory characteristics to avoid delayed diagnosis of SLE and to improve patient care.

\section{Han-You Mo, ${ }^{1,2}$ James Cheng Chung Wei $\odot,^{2,3,4}$ Xiao-Huan Chen $\odot{ }^{2,5}$ Hsin-Hua Chen ${ }^{6,7,8,9,10}$}

'Department of Rheumatology, The Affiliated Hospital of Guilin Medical University, Guilin, China

${ }^{2}$ Institute of Medicine, College of Medicine, Chung Shan Medical University,

Taichung, Taiwan

${ }^{3}$ Department of Allergy, Immunology \& Rheumatology, Chung Shan Medical University Hospital, Taichung, Taiwan

${ }^{4}$ Graduate Institute of Integrated Medicine, China Medical University, Taichung, Taiwan

${ }^{5}$ Department of Rheumatology, Guilin Medical University, Guilin, China

${ }^{6}$ Division of Allergy, Immunology and Rheumatology, Taichung Veterans General Hospital, Taichung, Taiwan

7Department of Medical Research, Taichung Veterans General Hospital, Taichung, Taiwan

${ }^{8}$ Department of Industrial Engineering and Enterprise Information, Tunghai

University, Taichung, Taiwan
${ }^{9}$ School of Medicine, National Yang-Ming University, Taipei, Taiwan

${ }^{10}$ Institute of Biomedical Science and Rong Hsing Research Centre for Translational Medicine, Chung Hsing University, Taichung, Taiwan

Correspondence to Dr Hsin-Hua Chen; shc5555@hotmail.com Handling editor Josef S Smolen

Contributors $\mathrm{H}$-YM and JCCW conceptualised the research and drafted the manuscripts. X-HC interpreted the data and drafted the manuscript. H-HC contributed to the research design, performed data analysis and graph generation, and critically revised the manuscript. All authors read and approved the final manuscript.

Funding This work was supported by funding from Chung Shan Medical University Hospital (grant number CSH-2018-C-023) and the National Natural Science Foundation of China (grant number 81760298).

Competing interests None declared.

Patient consent for publication Obtained.

Ethics approval This study was approved by the institutional review board of Taichung Veterans General Hospital in Taiwan (approval number CE17100B)

Provenance and peer review Commissioned; internally peer reviewed.

Supplemental material This content has been supplied by the author(s). It has not been vetted by BMJ Publishing Group Limited (BMJ) and may not have been peer-reviewed. Any opinions or recommendations discussed are solely those of the author(s) and are not endorsed by BMJ. BMJ disclaims all liability and responsibility arising from any reliance placed on the content. Where the content includes any translated material, BMJ does not warrant the accuracy and reliability of the translations (including but not limited to local regulations, clinical guidelines, terminology, drug names and drug dosages), and is not responsible for any error and/or omissions arising from translation and adaptation or otherwise.

(c) Author(s) (or their employer(s)) 2020. No commercial re-use. See rights and permissions. Published by BMJ.

- Additional material is published online only. To view, please visit the journal online (http://dx.doi.org/10.1136/annrheumdis-2020-219269).

H-YM and JCCW contributed equally.

\section{Check for updates}

To cite Mo H-Y, Wei JCC, Chen X-H, et al. Ann Rheum Dis Epub ahead of print: [please include Day Month Year]. doi:10.1136/annrheumdis-2020-219269

Received 19 October 2020

Accepted 20 October 2020

\section{Linked}

- http://dx.doi.org/10.1136/annrheumdis-2020-219257

Ann Rheum Dis 2020;0:1-2. doi:10.1136/annrheumdis-2020-219269

\section{ORCID iDs}

James Cheng Chung Wei http://orcid.org/0000-0003-0310-2769

Xiao-Huan Chen http://orcid.org/0000-0002-5482-4756

Hsin-Hua Chen http://orcid.org/0000-0002-7304-4587

\section{REFERENCES}

1 Huang H, Zhang ZL. Correspondence on: increased risk of systemic lupus erythematosus in patients with autoimmune haemolytic anaemia by Mo, et al. Ann Rheum Dis 2020.

2 Mo H-Y, Wei JCC, Chen X-H, et al. Increased risk of systemic lupus erythematosus in patients with autoimmune haemolytic anaemia: a nationwide population-based cohort study. Ann Rheum Dis 2020. doi:10.1136/annrheumdis-2020-218886. [Epub ahead of print: 22 Sep 2020].

3 Zhu FX, Huang J-Y, Qingqing W, et al. Response to: 'Risk of systemic lupus erythematosus in patients with idiopathic thrombocytopenic purpura: a need for a more accurate control group?' by Mertz and Arnaud. Ann Rheum Dis 2020. doi:10.1136/ annrheumdis-2020-217696. [Epub ahead of print: 20 May 2020].

4 González-Naranjo LA, Betancur OM, Alarcón GS, et al. Features associated with hematologic abnormalities and their impact in patients with systemic lupus erythematosus: data from a multiethnic Latin American cohort. Semin Arthritis Rheum 2016;45:675-83. 